\title{
Die mecklenburgische Kunstgeschichtsschreibung
}

\author{
Ein Beitrag zur Geschichte des regionalen Kunstschriftums
}

Auch in Mecklenburg fehlt wie in den anderen deutschen Landschaften bis jetzt eine Bibliographie zur Kunstgeschichte, jedoch wird die Sammlung und Sichtung des Schrifttums durch das Vorhandensein allgemeiner und geschichtlicher Bibliographien, von denen die frühesten bereits aus dem 18. Jahrhundert stammen, wesentlich erleichtert $(1-3)$. Diese geben lediglich eine nicht geordnete Aufzählung der erschienenen Titel. Erst das 19. Jahrhundert ordnet die Literatur nach sachlichen Gesichtspunkten und richtet das Hauptaugenmerk auf das geschichtliche und landeskundlich-topographische Schrifttum (4-8). Ihren umfassenden Abschluß finden diese Arbeiten in der ,Geschichtlichen Bibliographie von Mecklenburg “ von Wilhelm Heeß (10), einem Werk, das die geschichtliche Literatur Mecklenburgs von den Anfängen des Buchdrucks bis zum Jahre 1940 enthält. Heeß nennt die geschichtliche Literatur vollständig, das kunstgeschichtliche Schrifttum jedoch ist nur so weit erfaßt, wie es in den heimat- und landeskundlichen Zeitschriften und in den mecklenburgischen Kunstmonographien zur Verfügung stand. Auf die systematische Durchsicht der gesamten deutschsprachigen Kunstliteratur mußte selbstverständlich im Rahmen einer vorwiegend geschichtlichen Regional-Bibliographie verzichtet werden.

Diese Lücke sucht die vorliegende Bibliographie zur mecklenburgisch-vorpommerschen Kunstgeschichte zu schließen. Ein kurzer Überblick über die einzelnen Phasen der mecklenburgischen Kunstgeschichtsschreibung sei der eigentlichen Bibliographie vorangestellt, da er über die Besonderheiten der Entwicklung dieser Kunstgeschichtsschreibung Auskunft geben kann und die bestehenden großen Lücken in der Forschung deutlich werden läßt. Er beginnt mit den mittelalterlichen Historikern des 16. Jahrhunderts und versucht bis zur kunsthistorischen Forschung der Gegenwart kritisch vorzudringen.

\section{Die historischen Schriften}

a) Die mittelalterlichen Historiker

Schon früh berichten mittelalterliche Chroniken über den Charakter des Landes, über Lebensweise und Kleidung der Bewohner. Ibrahim Ibn Jacub schreibt in seinem Bericht über die Slaven aus dem Jahre 973 (585 u. 586) „Diese Burg heisst (Wili)-Grad, welcher Name „Grosser Berg“ bedeutet. Wili-Grad ist in einem Süßwassersee erbauet, wie die meisten Burgen der Slaven. Wenn sie nämlich eine Burg gründen wollen, so suchen sie ein Weideland, welches an Wasser- und Rohrsümpfen reich ist, und stecken dort einen runden und viereckigen Platz ab, je nach der Gestalt und dem Umfange, welche sie der Burg geben wollen" (585, S. 7). Die Chronik Thietmars von Merseburg ${ }^{1}$ aus der Zeit um 1000 und die Adams von Bremen ${ }^{2}$ aus der Mitte des 11. Jahrhunderts erwähnen nur Geschichte und geographische Beschaffen-

1 Gesch.-Schrn. d. dt. Vorzeit XI. Jh. Bd. 1

2 Gesch.-Schrn. d. dt. Vorzeit XI. Jh. Bd. 6 
heit der einzelnen Länder. In der Reimchronik des Ernst von Kirchberg (573, Sp. 583-814) aus dem 14. Jahrhundert wird bereits gelegentlich ein Kunstwerk genannt. Die Bemerkung über den Neubau der Doberaner Klosterkirche ,Im Appid ward vort an, von Dalym her Johan, der brach daz hulzene Münstir nider, und machte es schöne steynen wider" (573, Sp. 781), ist für die Baugeschichte von Doberan von größter Wichtigkeit und darüber hinaus eine der frühesten Quellen zur mecklenburgischen Kunstgeschichte.

b) Die Historiker des 16. Jahrhunderts

Nach der Mitte des 16. Jahrhunderts entstehen auch in Mecklenburg Geschichtswerke nach antikem Vorbild. Albert Krantz gibt in seinen Hauptwerken, der ,Wandalia“3 und der „Saxonia“4, eine Übersicht über die Geschichte der Vandalen und Sachsen. Als Historiker des Humanismus schreibt er seine Werke nach dem Vorbild antiker Annalen. Die Quellen sind die Urkunden der einzelnen Länder, die er für die Wandalia vor allen Dingen bei Blondus ${ }^{5}$, Aeneas Sylvius ${ }^{6}$ (dem späteren Papst Pius II.) und bei Saxo Grammaticus fand. ${ }^{7}$ Lateinische Annalen waren auch das Vorbild für die ,,Annalium Herolorum ac Vandalorum libri septem" von Nicolaus Marschalk (573, Sp. 166-562). Für die vorgeschichtliche Forschung ist dieses Geschichtswerk von besonderer Bedeutung, weil hier von der Stadt Rethra gesprochen wird: , , . . noch vielmehr in einer Stadt Rether, die lag etwan von Malchin nicht weit, was groß und mächtig bey der Zeit. Neun Pforten hatte die Stadt gar eben, mit einem tiefen Teich umgeben. Die Stadt hatte nur einen Ausgang ueber eine Brücke schmal und lang." 8

Historischen Charakter haben die Schriften von Johann Simonius (573) und David N. Chyträus (669).

\section{Die topographisch-historischen Schriften}

Daneben entstehen in dieser Zeit die Gesamtdarstellungen, die die Topographie des Landes in den Vordergrund stellen. Die früheste Darstellung dieser Art, ,,Mecklenburg“ von Sebastian Münster (568 u. 569, S. 575-577), geht allerdings nur ganz kurz auf Geographie und Geschichte des Landes ein, ohne Einzelheiten zu erwähnen. Anders in der rund 100 Jahre später erschienenen ,,Topographia Saxonia Inferioris“ von M. Zeiller (571). Hier sind in der "Beschreibung des Nieder-Sächsischen Craysses“ die einzelnen Städte alphabetisch geordnet und topographisch-historisch beschrieben. In dem Kapitel „Wismar“ heißt es u. a.: ,, . . und seyn da zu sehen S. Nicolaii, S. Mariae, S. Georgii, und des H. Geistes alles stattliche (sonderlich die 3. erste) Kirchen; Item das graue Closter/schwartz Closter/ und das Rathhaus ... Die Statt hat einen ansehenlichen Marckt/ oder Platz, und Weinkeller" (571, S. 238). Und von Rostock wird geschrieben: „Aus den erzehlten Kirchen, seyn vier Pharren, als zu St. Jacob, darauff ein stattlicher Thurm; St. Marien mitten in der Statt, ein prächtiges Gebäw, die zu S. Peter, und die S. Niclas" (571, S. 205).

Die einzelnen Gebäude, vor allem die Kirchen, werden lediglich aufzählend erwähnt, ohne daß man etwas über ihr Aussehen oder über Einzelheiten des Baues erfährt.

${ }^{3}$ Crantzii, A., Wandalia oder Beschreibung wendischer Geschichte . . übersetzt durch Stefan Macropum. Lübeck 1600 u. 1636.

4 Krantz, A., Saxonia. Weitleuffige ... Beschreibung der Ankunft, Sitten, Regiment . . der Sachsen verdeutscht durch Basilium Fabrum Soranum, Leipzigk 1563 u. 1582.

5 Nordmann, V. A., Die Wandalia des A. Krantz, S. $109 \mathrm{ff}$.

${ }^{6}$ Nordmann, a. a. O., S. 107

7 Nordmann, a. a. O., S. 111

8 Bützowsche Ruhestunden 18. 1765, 66 


\section{Reiseschilderungen}

Kunst- und kulturgeschichtlich ergiebiger als die topographisch-historischen Schriften sind die seit Ende des 16. Jahrhunderts entstandenen Reisebeschreibungen. Sie vermitteln nicht nur durch ihren Stil und durch die Auswahl des Beschriebenen ein kulturgeschichtliches Bild ihrer Zeit, sondern in ihnen werden häufig heute längst verschwundene Gebäude und Kunstgegenstände erwähnt. So wird das ursprüngliche Aussehen des herzoglichen Schlosses zu Schwerin in dem „Reisetagebuch des Herzogs P. J. von Pommern-Wolgast“ im Jahre 1602 (1078) folgendermaßen beschrieben: ,... und um die Vestung gefeuret ist, welche in einem grossen See, so in 4 Meilen lang, gelegen ist; ein wohl gebautes und mit verguldeten Gipsfiguren und künstl. Mahlwerck wohl geputztes Haus" (1078, S. 86). In diesem Tagebuch werden die kirchlichen und weltlichen Feste ausführlich erwähnt, die Kunstwerke jedoch nur soweit, als sie Merkwürdigkeiten darstellen. So heißt es von dem Schweriner Dom: „Im Dohm, so noch in der Stadt beybehalten wird, sind zwo fürstliche Begräbniße, ein altes Herzog Johann Albrechts, und ein neues von schwartzem und weißem Marmor Hertzog Christophels, welches über die 2000 Thaler soll gestanden seyn. Durch ein Fenster kan man die Leiche sehen, so mit einem langen schwartzen Sammet bedecket" (1078, S. 86).

Das Tagebuch der "Akademischen Ferienreise von Rostock bis Königsberg im Jahre 1694“ (1082, S. 1-54) führt die Innenausstattung der Kirchen eines Inventars auf. Der Verfasser Carl Arndt (Carolus Arndinus) schreibt von der Nikolaikirche in Stralsund: ,,a) Ein schön steinerne Cantzel. b) Ein schöner Taufstein nebst dem Opfertisch“ (1082, S. 5). Von St. Marien in Stralsund nennt er: ,, a) Ein schöner geschnitzter Engel mitten in der Kirche in Lebensgrösse so aber ein Weibesbild über ihr Grab in Spem resurrectionis futurae hatt setzen lassen" $(1082$, S. 6).

Die Schreiber des 16. und 17. Jahrhunderts beschränken sich zumeist nur auf die bloße Erwähnung des vorhandenen Bestandes, ohne durch die kritische Auswahl oder durch die besondere Hervorhebung einzelner Gebäude und Kunstwerke ein Verständnis für die künstlerische Bedeutung einzelner Objekte zu zeigen. Das Interesse an dem Kultur- und Gesellschaftsbild der eigenen Zeit, verbunden mit dem historisch-kritischen Verständnis für die Kunstwerke der Vergangenheit, finden wir erst bei den Reisenden des 18. Jahrhunderts.

Dr. Nugent beweist in seinen ,,Travels through Germany, with a Particular Account of the Courts of Mecklenburg, in a Series of Letters to a Friend" (1053 u. 1054) sowohl den Scharfblick für die politischen und soziologischen Fragen des Landes als auch eine - wenn auch natürlich zeitgebunden rationalistische - Urteilsfähigkeit in Fragen der Kunst. So schreibt er von der Marienkirche in Wismar: „Die Marienkirche ist ein feines gotisches Gebäude und hat einen sehr hohen Turm... Inwendig ist die Kirche nach lutherischer Art mit einer Menge von Gemälden und Monumenten ausgeziert; besonders das Gebäude ist sehr kunstreich“ (1092, S. 18). Sein rationalistischer Geschmack möchte jedoch alle Kirchen weiß übermalt haben, denn er meint: „, . . Am anstössigsten war's mir, dass die Pfeiler nicht einmal überweisst, sondern die puren Mauersteine zu sehen waren" (1092, S. 18).

Die Ende des 18. Jahrhunderts aufkommende Neigung der Engländer für die gotische Architektur spricht auch aus Nugents Worten über die Kirchen Rostocks: „Alle sind gotischer Bauart und mit Ausnahme der Marienkirche nicht im geringsten wegen ihrer Schönheit merkwürdig" (1092, S. 38). Erst recht mußte ihn daher die Klosterkirche in Doberan begeistern: „Die Kirche ist ein ehrwürdiges, gotisches Gebäude, hell und geräumig, und kann inmer für ein schönes Stück aus der alten Architektur gelten. Der Hochaltar ist noch ebenso als zu den Zeiten des Papsttums mit grotesken, hölzernen Figuren aufgeputzt. In dieser Kirche wird eine unzählbare Menge von Altertümern aufbewahrt, die in Gemälden, Statuen, Grabschriften, Inschriften und Reliquien bestehen" (1092, S. 45). Der Rostocker Marktplatz und das Rathaus werden in verschiedenen Reisebeschreibungen erwähnt. Friedrich Johann Zöllner schreibt in seiner ,Reise durch Pommern nach der Insel Rügen und einem Theile des Herzogthums Mecklenburg im Jahre 1795“ (1064):,, Der Markt ist ein ansehnlicher viereckter Platz, auf welchem in 
der mitte ein Brunnen und der Schandpfahl (Kahk) stehn. .. Die Häuser, welche den Marktplatz einschliessen, sind lauter Giebelhäuser ... Den grössten Theil der östlichen Seite des Marktes nimmt das Rathhaus ein. Der vorspringende, drei Stockwerk hohe Theil desselben, scheint erst in neueren Zeiten ausgebaut zu seyn: wenigstens sind die sieben Thürmchen, die sonst wol eine Zierde der ursprünglichen Facade waren, jetzt von vorn kaum sichtbar. Unten sind Schwiebbogen, worin Waaren verkauft werden" $(1064$, S. 384).

Wilhelm v. Humboldt vermerkt in seinem „Tagebuch von der Reise nach Norddeutschland im Jahre 1796“: „Aber der Markt, auf dem sich das Rathhaus auszeichnet, ist sehr gross . . “ (1081, S. 54). Die Abneigung des späten 18. Jahrhunderts gegen die Bewegtheit des Barock drückt sich in den Bemerkungen Zöllners über die Rostocker Marienkirche aus: „Die Marienkirche hat ein sehr hohes Gewölbe, in Form eines Kreuzes. Die Bildhauereien darin sind schlecht, und die Gemälde, womit das hohe Altar geschmückt ist, rühren von keinem sonderlichen künstler her. Aber einen prächtigen Anblick giebt das fürstliche Chor. Es ist weiß, mit einer reichen Vergoldung und wird von einem rothen Baldachin bedeckt. Ueber diesem Baldachin prangt die Orgel, deren Pfeifen in zwei schön geordneten Reihen über einander stehen. Wäre man weniger verschwenderisch mit dem Goldschaume gewesen: so würde das Ganze einen geschmackvolleren Eindruck machen, ohne an Pracht zu verlieren“ (1081, S. 395).

\section{Die gelehrten Zeitschriften}

Seit etwa 1720 erscheinen in allen deutschen Landen die gelehrten Zeitschriften. Während die periodischen Schriften des 17. sowie des 18. Jahrhunderts noch in lateinischer Sprache abgefaßt waren, werden die gelehrten Aufsätze teils erbaulichen, teils belehrenden Charakters in deutscher Sprache geschrieben. Sie sind vorwiegend ein Bildungsmittel für die geistig interessierten Schichten des Bürgertums. Wenn diese Zeitschriften auch noch reich sind an Schilderungen von kuriosen Begebenheiten, so sind ihre mehr oder minder breiten Abhandlungen doch meist historischen Inhalts. Historisch-biographisch ist auch das Interesse an der Kunst und den Künstlern. Diese neue Art der Betrachtung ist noch nicht frei von moralisierenden Zügen, doch bemüht man sich, den Künstler stärker als bisher aus seinem Werk zu verstehen. Die erste gelehrte Zeitschrift in Mecklenburg erscheint 1737 unter dem Titel „Etwas von gelehrten Rostockschen Sachen für gute Freunde“ (69 u. 70) und bringt allwöchentlich dem „geehrtesten Leser“ Artikel aus „,der einheimischen Geschichte der Gelahrtheit“, wie es in der Vorrede des ersten Heftes heißt. Man verspricht: „Und wird man sich bemühen, Lebens-Läuffe, programmata inauguralia introductoria, exequialia, Vitas aus den Leichen-Predigten, Catalogos Lectionum, Nachrichten von den Schrifften der Academischen und andern Gelehrten Rostockschen Männer, die Seriem der Professorum der Pastorum u. s. f. Imgleichen Erzehlungen von allerhand sunderbahren memorabilibus der Academie, Ihren Rechten, Factis, Veränderungen, alten und neuen Institutes und dergleichen, jedoch aus sicheren Grunde zu liefern. " 9 Diese Zeitschrift will also von der Universität und ihrem Leben berichten. Der erste Aufsatz mit der Überschrift „Die Nachricht von dem Ursprung der Universitaet Rostock" behandelt daher auch die Gründung der alma mater Rostochiensis.

An vorwiegend gelehrte Leser wenden sich auch die „Bützowschen Ruhestunden, gesucht in Mecklenburgschen vielentheils bisher noch ungedruckten zur Geschichte und Rechtsgelahrtheit vornehmlich gehörigen Sachen" (76). Wie schon der ausführliche Titel sagt, will man bis dato unveröffentlichte Urkunden und Akten dem „Gelehrten Publikum“ zugänglich machen. So haben die Aufsätze über die Bützower Kirche, über mecklenburgische Münzen und Wappen rein beschreibenden Charakter und veröffentlichen das Quellenmaterial ohne Kommentar.

Eine weitere gelehrte Zeitschrift, die „Gemeinnützigen Aufsätze aus den Wissenschaften für alle Stände, zu den Rostockschen Nachrichten“ (19), bringt in ihrem Jahrgang 1777 eine „Beschreibung der

๑ 69, Jg. 1, 1737 . 
Sct. Marienkirche hie in Rostock, nebst einem Verzeichnisz der seit der Reformation bis diese Zeit daran gestandenen Prediger “. Der Verfasser G. V. H. Niehenck erwähnt - und das ist typisch für diese Zeit von dem Inventar der Kirche nur den Barockaltar und die silbernen Abendmahlsgeräte, während die aus „papistischer Zeit" stammenden mittelalterlichen Altäre für den lutherischen Pastor überhaupt nicht existieren.

\section{Die Kunstgeschichtsschreibung des 19. Jahrhunderts}

Wie in allen deutschen Landen begann auch in Mecklenburg die systematische Erforschung und Darstellung der Landeskunst nach den Befreiungskriegen, als das Interesse an der vaterländischen Kultur und ihrer Entwicklung überall erwachte. In den $30 \mathrm{er}$ Jahren des 19. Jahrhunderts entstanden allerorts die Vereine zur Pflege der heimatlichen Kunst und Geschichte. Bereits 1824 wurde die „Gesellschaft für Pommersche Geschichte und Altertumskunde“ gegründet, 1833 folgte der ,Verein für Schleswig-Holsteinsche Geschichte“ und kurz darauf der unter ,,allergnädigster Protektion“ stehende „Verein für Mecklenburgische Geschichte und Altertumskunde". Bereits ein Jahr nach der Gründung erschien der erste Band der "Jahrbücher des Vereins, für Mecklenburgische Geschichte und Altertumskunde“. Der Herausgeber war der 1. Sekretär des Vereins, Georg Christian Friedrich Lisch, der auch die meisten Aufsätze verfaßte. Er schrieb über einzelne Kirchen, ihre Baugeschichte und ihre Ausstattung, über Siegel und Wappen, über die Lage slawischer und mittelalterlicher Burgen und über vorgeschichtliche Bodenfunde. Seine Arbeiten entstanden aus kritischer Durchsicht und Auswertung unveröffentlichter Urkunden und sind heute noch eine unerschöpfliche Quelle für die mecklenburgische Geschichte und Kunstgeschichte. Allerdings sind seine Aufsätze, der wissenschaftlichen Methode des 19. Jahrhunderts entsprechend, meist nicht wertenden, sondern beschreibenden Charakters. G. C. F. Lisch hat das unbestrittene Verdienst, die mecklenburgische Geschichtsschreibung aus dem Zusammenhang mit naturkundlichen und landwirtschaftlichen Erörterungen gelöst zu haben. Die „Mecklenburgischen Jahrbücher“ sind die erste wirklich kulturgeschichtliche Zeitschrift. In ihnen ist kein Platz mehr für Aufsätze über Bodenbeschaffenheit und Viehkrankheiten, die noch in dem gleichzeitig erscheinenden „Archiv für Landeskunde" und in dem „Freimütigen Abendblatt" ebenbürtig neben der Beschreibung einer Kirchenglocke standen.

Nach dem Tode G. C. F. Lischs im Jahre 1883 übernahm Friedrich Wigger die Herausgabe der Jahrbücher, jedoch blieb unter seiner und seines Nachfolgers, Grotefend, Leitung die von Lisch vorgezeichnete Linie weiterhin bestimmend. Allerdings war Grotefend vorwiegend Historiker und hatte kein so ursprüngliches Verhältnis zur mecklenburgischen Kultur. Aus diesem Grunde lag das Schwergewicht mehr auf der Veröffentlichung historischer Urkunden und Akten. Neben G. C. F. Lisch bestimmte Friedrich Schlie das Gepräge der mecklenburgischen Kunstliteratur des 19. Jahrhunderts. Er war zwar historisch nicht so umfassend gebildet wie Lisch, aber sein kunstgeschichtliches Wissen war umfangreicher. Er hatte Klassische Philologie und Archäologie studiert (111), war dann Hilfssekretär am Archäologischen Institut in Rom gewesen und hatte 1877 die Leitung, ein Jahr später die Oberleitung der Schweriner Kunstsammlungen übernommen. Sein Hauptinteresse galt der Gemäldegalerie. 1882 erschien sein „Beschreibendes Verzeichnis der Werke älterer Meister in der Großherzoglichen Gemälde-Gallerie zu Schwerin" (218). Er arbeitete aber auch über einzelne Kunstwerke in mecklenburgischen Kirchen. Er schrieb Aufsätze wie „Die eherne Fünte von St. Marien zu Rostock“ (3217), „Das Altarwerk der Brüsseler Meister Jan Borman und Bernaert van Orley in der Pfarrkirche zu Güstrow" (2575) und der "Altarschrein in der Stadtkirche zu Grabow, kein Lübecker, sondern ein Hamburger Werk" (2429), um nur einige seiner bekanntesten Publikationen zu nennen. Schlies Name wurde jedoch nicht so sehr durch diese Veröffentlichungen über die Grenzen Mecklenburgs hinaus bekannt, sondern durch die Herausgabe der „Kunstund Geschichtsdenkmäler des Herzogthums Mecklenburg-Schwerin" (80). In diesem mecklenburgischen Inventar stellt Schlie, der wissenschaftlichen Auffassung seiner Zeit folgend, die historischen Fakten in 
den Vordergrund seiner Untersuchungen. Die Stammbäume der Herzöge und die Namensregister der Pastoren überwuchern an manchen Stellen die kunstgeschichtlichen Ausführungen. Auch zeigt sich immer wieder Schlies Studium der Klassischen Philologie in den philologischen und ikonographischen Untersuchungen. Aber trotzdem sind die Baubeschreibungen mit exakt-kunsthistorischer Methode vorgenommen. Wenn auch "der Schlie“ in seinen wissenschaftlichen Ergebnissen heute überholt ist, so ist er doch durch sein reiches Quellen- und Abbildungsmaterial ein unentbehrliches Hilfsmittel.

\section{Die Kunstgeschichtsschreibung von 1900 bis zur Gegenwart}

Während des 19. Jahrhunderts blieb die Kunstgeschichte meist im Schatten der allgemeinen Geschichte. Ihre Verselbständigung als Wissenschaft vollzog sich entscheidend erst um die Jahrhundertwende, als Gelehrte wie Anton Springer und Georg Dehio in ihren Darstellungen das Bild der deutschen kunstgeschichtlichen Entwicklung zeichneten, das in seinen Grundzügen noch heute Bestand hat.

In der mecklenburgischen Kunstgeschichtsschreibung hatte bereits Friedrich Schlie mit seiner Arbeit über „Eine Kanne des Christoph Jamnitzer im Schweriner Museum“ den neuen Weg der exakten Stilbestimmung beschritten (220). Aber der jüngeren Generation blieb es vorbehalten, diese Arbeitsmethoden auszubauen.

Mit der Gründung der kunstgeschichtlichen Lehrstühle an den deutschen Universitäten erwachte auch das Interesse an den landschaftlich gebundenen Leistungen der Architektur, Plastik und Malerei. Die erste Dissertation über ein Thema der mecklenburgischen Kunstgeschichte legte Friedrich Sarre 1890 der philosophischen Fakultät Leipzig unter dem allgemein lautenden Titel ,Beiträge zur mecklenburgischen Kunstgeschichte" (2183 u. 2184) vor. Ausgehend von der Tätigkeit des Lübecker Ziegelformers, Statius von Düren, am Fürstenhof in Wismar, behandelt er die mecklenburgische Renaissance-Architektur zwischen $155^{\circ}$ und 1600 . Aber auch Sarre ist der Betrachtungsweise des 19. Jahrhunderts noch stark verhaftet. Er bringt im 2. Teil seiner Arbeit lediglich eine registrierende Aufstellung der „,Künstler und Werkmeister in Mecklenburg von 1550-1600" nebst Briefanlagen. Er setzt also historische und archivalische Nachrichten noch gleichwertig neben die Werkanalyse. Eine Deutung und vor allem Wertung der künstlerischen Leistung findet man hier nicht.

Diese persönliche Wertung gibt erst der Dehio-Schüler Heinrich Reifferscheid in seiner Dissertation „Der Kirchenbau in Mecklenburg und Neuvorpommern zur Zeit der deutschen Kolonisation“ (1469). Wie Georg Dehio in seiner ,Geschichte der Baukunst“ und in seinen „Handbüchern der Deutschen Kunstdenkmäler" nimmt auch Reifferscheid - nachdem er die einzelnen Kunstdenkmäler beschrieben hat - zu ihnen Stellung und urteilt über Qualität und entwicklungsgeschichtliche Bedeutung der verschiedenen mecklenburgischen und vorpommerschen Kirchen.

Von allen Gebieten der mecklenburgischen Kunst war die Architektur zuerst Gegenstand kunsthistorischen Forschens. Das ist ganz erklärlich, weil die Baukunst von der Spätromanik bis zum Klassizismus die bedeutendsten Leistungen der landschaftlichen Kunst hervorgebracht hat.

Nach der Errichtung der kunstgeschichtlichen Seminare an den Universitäten Rostock (1919) und Greifswald (1906) wurde die mittelalterliche Baukunst Mecklenburgs und Vorpommerns in zahlreichen Einzeluntersuchungen dargestellt.

Diese Reihe beginnt mit der Dissertation von Leonie Reygers über ,Die Marienkirche in Bergen auf Rügen und ihre Beziehungen zur dänischen Backsteinarchitektur" (1506). Die Verfasserin kommt nach einer kritischen Beschreibung und Würdigung des Hauptbaues zur stilistischen Verbindung der Marienkirche mit dänischen Kirchen und hat durch das Nachweisen dieser Beziehungen die Frage nach der Herkunft der Baukunst Rügens allgemein weitgehend gelöst. Methodisch ähnlich geht auch Albrecht Volkmann in seiner Arbeit „Das Kloster Sonnenkamp zu Neukloster in Mecklenburg“ (1742 u. 1743) vor. Durch die Grabungen des Instituts für Kunstgeschichte Rostock konnte er den Hauptbau sehr genau datieren. In dem Vorspann seiner Dissertation über die Anfänge der Backsteinbaukunst kommt Volkmann 
allerdings zu recht anfechtbaren Ergebnissen. Trotzdem muß diese Veröffentlichung für die mecklenburgische Architekturforschung als mustergültig bezeichnet werden.

Otto Francke beginnt seine Dissertation über ,,St. Nicolai zu Rostock. Die erste chorlose Hallenkirche in Mecklenburg“ (1858) mit einer Baubeschreibung. Dann kommt er zu den westfälischen Vorstufen der chorlosen Hallenkirche und schließt an diese die typverwandten Kirchen Mecklenburgs an. Trotz neuer Einzelerkenntnisse zum Problem der frühen Hallenkirchen in Mecklenburg fehlt der Franckeschen Arbeit die größere Zusammenschau dieses Komplexes. Die 1940 erschienene Arbeit von Ludwig Rohde „St. Marien zu Greifswald und die frühe Backsteingotik im wendischen Quartier der Hanse" (1624) ist schlechthin vorbildlich für die Darstellung der frühgotischen Architektur im norddeutschen Raum. Methodisch geht Rohde ähnlich vor wie Francke und beginnt seine Untersuchungen mit einer Baubeschreibung. Nach der stilgeschichtlichen Einordnung der einzelnen Bauteile kommt er zur eigentlichen Geschichte des Baues und schließlich zur kunstgeschichtlichen Stellung der Greifswalder Marienkirche innerhalb der vorpommerschen und mecklenburgischen Baukunst um 1250.

Die Dissertation von Wolfgang Clasen ,,St. Marien zu Stralsund und der Kirchenbau der Spätgotik im norddeutschen Backsteingebiet" (1939) behandelt eine Epoche der norddeutschen Baukunst, die bisher weniger als die Architektur des 13. und 14. Jahrhunderts Gegenstand des forschenden Interesses war.

Mit der spätmittelalterlichen Backsteinbaukunst beschäftigt sich auch die Untersuchung von Nikolaus Zaske „Herkunft und Bedeutung der Architektur Heinrich Brunsbergs“ (2089, 2090 u. 2090 a).

Typengeschichtliche Untersuchungen des mittelalterlichen Kirchenbaues sind die Dissertationen von Hugo Westphal „Die gotischen Landkirchen auf Rügen“ (1467) und von Joachim Fait „,Die Bettelordenskirchen zwischen Elbe und Oder" (1452). Die Arbeit von Westphal ist leider nur ein erweiterter Werkkatalog. Die Darstellung der Bettelordensarchitektur von Fait dagegen bringt eine gründliche Ubersicht über dieses bisher nur durch kleinere lokal begrenzte Einzeluntersuchungen (1993-1995, 1997, 1999) bekannte Gebiete.

Teilfragen der mittelalterlichen Profanbaukunst behandeln die Arbeiten von Jenny Müller ,,Mecklenburgische Stadttore“ (1382) und Theodor Straub „Mittelalterliche Backsteingiebel im Profanbau der Hansestädte des wendischen Kreises" (1429). Jenny Müller untersucht die mecklenburgischen Stadttore des 13. bis 15. Jahrhunderts, sie beschränkt sich auf die Sammlung des Materials und unternimmt den Versuch einer Stilkritik. In der Dissertation von Heinrich Trost „Norddeutsche Stadttore zwischen Elbe und Oder", 1959 im Druck erschienen (1383), werden die Stadttore Mecklenburgs, Brandenburgs und Vorpommerns einer genauen historisch-urkundlichen und stilkritischen Analyse unterzogen. Die Arbeit von Straub bringt lediglich eine Beschreibung der in den verschiedenen Städten des wendischen Kreises vorkommenden Giebelformen.

Die Dissertation von Herbert Möller über „Das mittelalterliche Kirchenportal in Vorpommern“ (1432) ist nach den einzelnen Elementen der Kirchenportale gegliedert. Sie registriert mehr den Bestand, als daß sie den Blick für die großen Zusammenhänge der Bauplastik im Backsteingebiet öffnet. Die Arbeit von Wolfgang J. Müller ,,Mittelalterliche Backsteinornamentik in Mecklenburg“ zeichnet sich durch ihre ungewöhnlich reiche Materialsammlung aus (1434). Sie vermittelt einen umfassenden Überblick über die mecklenburgische Baukunst des 13. Jahrhunderts und die Herkunft der Ziegelornamentik. Sie ist dadurch ein unentbehrliches Hilfsmittel für alle weiteren Forschungen der frühgotischen Architektur Norddeutschlands.

Die romanische Baukunst Mecklenburgs, ihre Abhängigkeit von Westfalen und Lübeck sowie ihre zeitliche Stellung in der Entwicklung Norddeutschlands wird weitgehend von Wolfgang Teuchert in seiner Dissertation „Die Baugeschichte der Petrikirche zu Lübeck“ (1705) geklärt. Vor allem bringt Teuchert neue Errgebnisse zur Datierung der Stadtkirche in Gadebusch, deren typenmäßige Abhängigkeit von der Petrikirche in Lübeck er nachweisen kann.

Aber nicht allein die Rostocker und Greifswalder Dissertationen brachten neue Forschungsergebnisse zur mittelalterlichen Baukunst Mecklenburgs und Vorpommerns, auch kleine Publikationen helfen 
Teilfragen lösen. Der Aufsatz ,,St. Jakobi zu Rostock und die Kathedralgotik Englands“ von Richard Sedlmaier, dem langjährigen Ordinarius für Kunstgeschichte an der Universität Rostock, stellt zum ersten Mal die Frage nach dem Einfluß der englischen Kathedralgotik auf einzelne Bauten des Küstengebietes (1835). In einer weiteren Arbeit über Backsteinbaukunst des mecklenburgischen Raumes (1116) gibt Sedlmaier einen skizzenhaften Ưberblick über die Entwicklung dieses Gebietes. Wenn es auch im Rahmen dieses Zeitschriftenaufsatzes nicht möglich war, neue Gesichtspunkte kunstgeschichtlichen Forschens zu vermitteln, so muß doch die klare und zusammengefaßte Aussageform als vorbildlich angesprochen werden.

Weitere Veröffentlichungen über die mecklenburgische Baukunst, wie die Arbeit von Karl Schmaltz „,Die Kirchenbauten Mecklenburgs“ (1185) und in jüngster Zeit das Buch von K. H. Clasen ,,Die Baukunst der Ostseeküste zwischen Elbe und Oder" (1096), bringen keine neuen Ergebnisse, sondern geben nur eine Übersicht über die Entwicklung der landschaftlich gebundenen Architektur von den Anfängen im 13. Jahrhundert bis zum Ende des Klassizismus.

Schmaltz hat dabei das Verdienst, die verschiedenen Typen des mecklenburgischen Kirchenbaues zum ersten Mal geordnet und die zeitliche Abfolge der Sakralbauten dargestellt zu haben. Heute sind freilich seine Thesen, besonders seine Meinung von dem ausschließlichen Einfluß der westfälischen Kirchen auf die frühen mecklenburgischen Bauten, durch spätere Einzeluntersuchungen überholt. Als Quellengrundlage wird jedoch das reiche Material dieses Buches seine Bedeutung behalten.

Clasen dagegen beschränkt sich nicht nur auf die kirchliche Architektur, sondern sieht die gesamte mecklenburgisch-vorpommersche Baukunst von 1200 bis 1830 in engem Zusammenhang mit der geschichtlichen und wirtschaftlich-soziologischen Entwicklung.

Die mecklenburgische Renaissancebaukunst ist schon um die Jahrhundertwende Gegenstand forschenden Interesses. Die Arbeit von August Hahr ,Die Architektenfamilie Parr“ (2204 u. 2206) ist allerdings ihrem biographischen Charakter nach noch der Betrachtungsweise des 19. Jahrhunderts verhaftet. Oskar Gehrig dagegen hat in seiner Dissertation „Philipp Brandin. Eine biographische Studie über den 1563-1594 tätigen Hauptmeister der mecklenburgischen Renaissance" (2197) zuerst einen Ubberblick über die kulturelle Situation Mecklenburgs nach der Reformation gegeben, um dann - wie es bei einer Künstlermonographie notwendig ist - die Lebensdaten Brandins anzuführen und schließlich die einzelnen Perioden seines Schaffens in Mecklenburg zu umreißen. Die Bedeutung dieser Arbeit liegt vor allem in der Publizierung bisher unbekannten, z. T. in dänischen und niederländischen Archiven aufbewahrten Materials.

Die mecklenburgische Baukunst des 18. und frühen 19. Jahrhunderts hat in den Bauten des Landadels und den Stadtgründungen der Herzöge einige bedeutende Leistungen hervorgebracht. Die mecklenburgischen Guts- und Herrenhäuser des 17., 18. und frühen 19. Jahrhunderts werden in der reichbebilderten Publikation von Jürgen Brandt „Alt-Mecklenburgische Schlösser und Herrensitze“ (2102) zum ersten Mal einem größeren Leserkreis zugänglich gemacht. Der Wert dieses Buches liegt daher auch weniger in der Vermittlung kunsthistorischer Ergebnisse, sondern vielmehr in seiner Zusammenstellung der im Lande verstreut liegenden und meist schwer erreichbaren Bauten der mecklenburgischen Profanarchitektur von der Spätrenaissance bis zum Klassizismus.

Die Haupt- und Nebenresidenzen der mecklenburgischen Herzöge, vor allem die Gründung des 18. Jahrhunderts, Ludwigslust, sind durch ihre architektonische Geschlossenheit mehrfach von der Forschung untersucht und dargestellt worden.

Johann Paul Dobert hat über „Bauten und Baumeister in Ludwigslust“ (2259) und Gerd Dettmann über ,Johann Joachim Busch, der Baumeister von Ludwigslust" (2199) geschrieben.

„Die Bauten des Seebades Doberan-Heiligendamm um 1800 und ihr Baumeister Severin“ (2244) behandelt die Dissertation von Hans Thielcke. Als Architekt bringt der Verfasser viele Rekonstruktionsund Planzeichnungen. Der Wert dieser Arbeit liegt in erster Linie in der Veröffentlichung bisher unbekannter Entwürfe. Die kunsthistorischen Zusammenhänge, die Frage nach der Herkunft Theodor Severins dagegen, werden nur am Rande gestreift. 
Severins Zeitgenosse David Anton Kuhfahl hatte zwar nicht diese Bedeutung wie jener, jedoch einen entscheidenden Einfluß auf die Profanarchitektur Güstrows. Lutz Köhlert hat in seiner Dissertation ,,Die klassizistischen Bürgerbauten in Güstrow und der Maurermeister David Anton Kuhfahl“ (2217 u. 2218) diesen Baumeister erstmalig mit einer kunsthistorischen Untersuchung gewürdigt. Die mecklenburgische Wohnhausarchitektur untersucht Rudolf Parow-Souchon (1225). Er hat das Material typengeschichtlich geordnet und behandelt, die einzelnen Bauten nur registrierend, so da $\beta$ wir eine Gesamtschau und eine Würdigung der norddeutschen Stadtbaukunst um 1800 vergeblich suchen.

Die Bauleistungen des 19. und 20. Jahrhunderts in Mecklenburg und Vorpommern haben bisher nur in Einzelfällen eine Behandlung erfahren. Jeder Versuch, das in Frage kommende Material wissenschaftlich zu durchdringen, ist bisher unterblieben. Da der landschaftsgebundene Charakter allgemein in diesen eineinhalb Jahrhunderten zurücktritt und die Bauwerke dieses Gebietes nur wenige hervorragende Leistungen aufweisen, ist diese Zurückhaltung der Forschung verständlich, doch wäre zu hoffen, daß eine Beschäftigung mit größerem Interesse einsetzt.

\section{Plastik}

In der Zeit von 1250 bis 1400 entstanden nicht nur die bedeutendsten Bauten der mittelalterlichen Sakralarchitektur Mecklenburgs, sondern im Zusammenhang mit der Architektur auch die wichtigsten Werke der Architekturplastik und im größeren Maße die Innenausstattung der Kirchen an Altären, Gestühlen und Andachtsbildern. Aus diesem Grunde beschäftigen sich auch die Arbeiten über die mecklenburgische Plastik vorwiegend mit den früh- und hochgotischen Werken bis zum Ausgang des weichen Stils.

Bereits im Jahre 1914 erschien die erste Dissertation über Probleme der mecklenburgisch-vorpommerschen Malerei und Plastik. Der Verfasser Max Paul nannte seine Forschungen ,,Sundische und Lübische Kunst" (2343) und behandelte neben Fragen der Stralsunder Wandmalerei, Werkstattprobleme der Stralsunder und Rostocker Plastik des weichen Stils. Leider erschöpft sich Pauls Arbeit im wesentlichen mit der Beschreibung der einzelnen stilverwandten Werke und mit der Abgrenzung der Werkstattkreise. Er setzt die Hegemonie der lübischen Kunst auch für das frühe 15. Jahrhundert noch voraus und kommt daher sowohl bei der Datierung als auch in der Würdigung der eigenständigen norddeutschen Plastik zu falschen Schlüssen.

Die Dissertation von Ernst Schneider ,,Schnitzaltäre des 15. und frühen 16. Jahrhunderts in Pommern" (2360) hat zwar das Verdienst, die pommersche Plastik nahezu vollständig erfaßt und chronologisch geordnet zu haben, kommt aber über eine nur katalogartige Aufzählung des Bestandes nicht hinaus. Eine geographische und stilistische Gesamtsicht der spätmittelalterlichen Schnitzkunst Pommerns sucht man hier vergebens.

Das Handbuch von Wilhelm Pinder „Die deutsche Plastik vom ausgehenden Mittelalter bis zum Ende der Renaissance" 10 hat auch auf die mecklenburgische Plastikforschung seit 1920 gewirkt. Margarethe Brückner ordnet in ihrer Publikation „Die Holzplastik in Mecklenburg von ihren Anfängen bis zum Ausklang des ,weichen Stils‘ (ca. 1250-1450)“ (2350) die plastischen Werke nach den von Pinder eingeführten Stilstufen. Allerdings werden in dieser Arbeit die einzelnen Plastiken nur beschrieben und nicht in geschichtliche und landschaftliche Zusammenhänge eingefügt. M. Brückner hat jedoch das Verdienst, erstmalig die einheimische mecklenburgische Kunst zu würdigen und ihre Selbständigkeit gegenüber Lübeck zu betonen.

Im Gegensatz zu M. Brückner glaubt Hellmuth Bethe in seiner Dissertation „Die mecklenburgischen Schnitzaltäre von der Mitte des 14. bis zur Mitte des 15. Jahrhunderts“ (2361) immer noch an die lübische Herkunft der bedeutendsten Plastiken in Mecklenburg. In seinem in der Zeitschrift „Nord-

10 Potsdam Wildpark, 1919. 
elbingen“ erschienenen Aufsatz: „,Lübische Schnitzaltäre der ersten Hälfte des 15. Jahrhunderts in Mecklenburg“ (2362) schreibt er "Mecklenburg besitzt damals noch keine Bildschnitzerwerkstätten von Ruf . .." (2362, S. 483).

Die Frage, wo die plastischen Werke entstanden sind, ob im Lande selbst oder in den westlichen Nachbargebieten, beschäftigt auch Berthold Conrades in seiner umfassenden und gründlichen Untersuchung "Niedersächsische und Hanseatische Plastik um 1400" (2346). Conrades geht allen vorher erschienenen Plastik-Publikationen gegenüber neue Wege. Er weist zwar auf stilistische Strömungen von Westfalen und Niedersachsen hin, betont aber die Selbständigkeit der mecklenburgischen Plastik.

Ein Einzelproblem der mecklenburgischen Plastik behandelt der Aufsatz von Friedrich Adolf Martens „Der Dreikönigsaltar der ehem. Johanniskirche zu Rostock“ (2502). Er bringt neue Ergebnisse über die sogenannte Rostocker Werkstatt. Martens setzt sich vor allem gründlich mit den Hypothesen der früheren Forschung und ihren oft spekulativen Datierungen auseinander. Sein Verdienst ist es, in dieser knappen Arbeit einen überzeugenden Beitrag zur Ausbreitung und zeitlichen Stellung der Rostocker Werkstatt gegeben zu haben.

In neuerer Zeit hat sich Ursel Schönrock noch einmal mit der mittelalterlichen mecklenburgischen Plastik beschäftigt. In ihrer Dissertation „Die Plastik in Mecklenburg von 1250-1350" (2351) gliedert sie das Material nicht chronologisch, sondern ordnet die Bestände im wesentlichen ikonographisch. Die Verfasserin dehnt den geographischen Begriff ,,Mecklenburg“ auch auf das Gebiet Vorpommerns mit Stralsund aus und kann dadurch für die frühgotische Plastik neue Verbindungen herstellen.

Ein wichtiges Werk der mittelalterlichen Plastik Mecklenburgs, den Doberaner Lettneraltar, untersucht Jens Christian Jensen in seiner Dissertation ,,Meister Bertram als Bildschnitzer. Das Verhältnis des Doberaner Lettneraltares zu den Skulpturen des Hamburger Petrialtars" (2573). Ausgehend von den Urkunden und archivalischen Nachrichten, beschäftigt sich Jensen mit dem Anteil Meister Bertrams an der Plastik des Lettneraltars. Als Schüler Walter Paatz' geht der Verfasser sehr gründlich vor und glaubt an dem Doberaner Schrein fünf verschiedene Schnitzer nachweisen zu können.

Wenn auch die künstlerisch bedeutendsten und ikonographisch interessantesten Werke der mittelalterlichen mecklenburgischen Plastik der Zeit von 1250 bis $145^{\circ}$ entstammen, so entstanden doch in der folgenden spätgotischen Epoche einige ausdrucksvolle Zeugnisse norddeutscher Schnitzkunst. Zu diesen gehören vor allem die Güstrower Domapostel und einige ihnen stilverwandte Werke. Da man seit Karl Schaefers Arbeit über Claus Berg (2549) annahm, daß die Güstrower Apostel eigenhändige Werke Bergs darstellen, sind sie immer wieder Gegenstand kunsthistorischer Erörterungen gewesen.

Die Anwesenheit Claus Bergs in Mecklenburg und seine eigenhändige Arbeit an den Domaposteln hat Hermann Deckert in seiner Dissertation „Die lübisch-baltische Skulptur am Anfang des 16. Jahrhunderts“ (2345) vorgeschlagen. In sprachlich ausgewogener Form bringt Deckert neue Forschungsergebnisse zur spätmittelalterlichen norddeutschen Plastik. Von den Arbeiten Claus Bergs ausgehend, wendet sich der Verfasser dann auch der anonymen lübischen Skulptur des frühen 16. Jahrhunderts zu. Diese Arbeit besticht sowohl durch die Flüssigkeit ihres Stils als auch durch die Kühnheit neuer stilkritischer Gesichtspunkte.

Während Deckert die Domapostel für eigenhändige Arbeiten Claus Bergs hält, schreibt Walter Josephi die Figuren einem Gehilfen und Schüler Claus Bergs zu. Er legt seine Auffassung kunsthistorisch zwar überzeugend, historisch aber durchaus anfechtbar in seinem kleinen Aufsatz ,Die Güstrower Domapostel“ (2439 u. 2441) dar und begründet seine Meinung mit der stilistischen Verschiedenheit zwischen dem Odenser Altar und den Güstrower Figuren.

Eine Gesamtdarstellung der spätmittelalterlichen Plastik Mecklenburgs, vor allem der Jahrzehnte von 1470 bis 1520, ist zum ersten Mal in der Dissertation von Edith Fründt ,Mecklenburgische Plastik von 1400 bis zum Ausgang des Mittelalters" (2353) versucht worden. Diese Arbeit soll einen Bestandsüberblick über die Werke vom Anfang des weichen Stils bis zum Ende der Spätgotik geben. Neben einer beschreibenden Würdigung der einzelnen Altäre, Triumphkreuzgruppen, Taufbecken, Grabplatten und Einzel- 
figuren geht die Verfasserin auch auf die ikonographischen Zusammenhänge innerhalb der norddeutschen Plastik ein. In einem angefügten Werkverzeichnis sind die besprochenen Arbeiten katalogartig aufgeführt und die Forschungsergebnisse in Stichworten angegeben.

Ein Randgebiet der mittelalterlichen Plastik untersucht A. Mehnert in ihrer Dissertation „Die mittelalterlichen Taufsteine in Pommern" (2391). Die Verfasserin ordnet das bisher unbekannte und unveröffentlichte Material typengeschichtlich und geht vor allem auf die Ornamentformen der Taufen ein, die durch die verschiedenen Gesteinsarten bedingt sind.

Die größeren Publikationen über die nachmittelalterliche Plastik beschäftigen sich vornehmlich mit der Skulptur des späten 16. und des 17. Jahrhunderts. Die Arbeiten von H. Hoops „,Die RenaissanceEpitaphien in Lübeck und Mecklenburg“ (2383) und von Ilse Römer ,Renaissanceplastik in Neu-Vorpommern" (2358) registrieren den Bestand. Darüber hinaus versuchen beide Verfasser, Stil- und Werkstatt-Gruppen zu finden.

Die Plastik des 18. Jahrhunderts wurde bisher nur in zwei Veröffentlichungen behandelt. G. Stades Dissertation über die „Mecklenburgischen Kanzelaltäre“ (2367 u. 2368) beschäftigt sich mit einer aus der lutherischen Liturgie erwachsenen Sonderform der Kanzel, die im 18. Jahrhundert zu einigen bemerkenswerten Erzeugnissen der Holzplastik geführt hat. „,Die Stralsunder Bildhauerkunst des 18. Jahrhunderts" (2613) hat K. Möller zum ersten Mal zusammenfassend dargestellt. Dabei kommt er zu interessanten Ergebnissen über die Rokokoausstattung von St. Nikolai in dieser Stadt.

Die Plastik des 19. Jahrhunderts ist von der Forschung bisher völlig übergangen worden. Vielleicht darf man sogar sagen, daß hierzu eine innere Berechtigung vorlag. Erst die Gestalt Ernst Barlachs, der durch sein Zurückziehen in die Umgebung von Güstrow gleichsam zu einem mecklenburgischen Bildhauer wurde, hat die Forschung wieder beschäftigt. Der Kunstkritiker Karl Scheffler wertete 1902 als erster in einem in der „Dekorativen Kunst“ erschienenen Aufsatz (2665) die Kunst dieses Meisters. Aber die wachsende Beachtung, die Barlachs plastisches und graphisches Werk erfuhr, war der Anlaß zu immer zahlreicher werdenden Aufsätzen, die in den $20 \mathrm{er}$ Jahren kritisch oder analysierend den Bildhauer Barlach und sein Werk durchleuchteten. Da Ernst Barlach von den Nazis verfemt wurde, lag von 1937 bis 1945 ein großes Schweigen über seinem Werk. Erst in den letzten 15 Jahren konnten sein Gesamtschaffen und seine Persönlichkeit voll gewürdigt werden.

\section{Malerei}

Die Arbeiten über die Malerei in Mecklenburg nehmen im Vergleich zu den Veröffentlichungen über die Architektur und Plastik nur einen kleinen Raum ein. Die mittelalterlichen Wandmalereien sind zum größten Teil zerstört, durch Restaurierungen entstellt oder noch nicht freigelegt. Die Tafelmalerei hat auf den Flügelaltären des weichen Stils einige bemerkenswerte Leistungen hervorgebracht. Jedoch ist in diesen Flügelmalereien der Einfluß der niedersächsisch-hamburgischen Schule so vorherrschend, daß man nicht von einem spezifisch mecklenburgischen Stil sprechen kann. Die mecklenburgische Altarmalerei war mehr als die gleichzeitige Plastik von äußeren Einflüssen abhängig, eine Eigentümlichkeit, die sich bis ins 16. Jahrhundert hinein verfolgen läßt. Sie ist daher auch nur im Zusammenhang mit der nordwestdeutschen Malerei erforscht worden.

Die Malerei der Renaissance und des Barock hat in Mecklenburg nur ein sehr begrenztes Aufgabengebiet. Das Deckengemälde in Kirche und Schloß fehlt vollständig, die dekorative Wandmalerei der Supraporten ebenfalls. Das Porträt war das einzige Anliegen der Rokoko- und Louis-seize-Malerei in Mecklenburg. In den Repräsentationsbildnissen Matthieus und Denners sind Adel und Bürgertum des Landes verewigt. Beide Maler haben nur provinzielle Bedeutung gehabt und sind, wie die Kirchen- und Historienmaler des 19. Jahrhunderts Suhrlandt und Lenthe, außerhalb der Grenzen kaum bekannt.

Die mittelalterliche Wandmalerei des Landes hat zuerst W. Burmeister bearbeitet. In seiner Dissertation „Wandmalerei in Mecklenburg bis 1400“ (2739) gibt er eine nach geographischen Gesichtspunkten 
geordnete Bestandsaufnahme der noch in Mecklenburg erhaltenen Wandmalerei. Leider ist der methodische Weg nicht klar genug herausgearbeitet, so daß die Werke wohl zeitlich geordnet werden, im übrigen aber beziehungslos nebeneinander stehen. Die mittelalterliche vorpommersche, insbesondere die Stralsunder Wandmalerei hat nach einem ersten Versuch durch Max Paul (2343) in E. Berckenhagen ihren gründlichen Bearbeiter gefunden. In seiner Dissertation „Die mittelalterliche Wandmalerei in Stralsund und im westlichen Pommern mit einem Beitrag zum Meister Bertram-Problem" (2742) gibt Berckenhagen nicht nur eine Zusammenstellung des Materials, sondern geht ausführlich auf die stilistischen und ikonographischen Zusammenhänge ein.

„Die Tafelmalerei des nordostdeutschen Küstengebietes von ihren Anfängen bis $145^{\circ}$ (2745) behandelt die Arbeit von F. A. Martens. Der Verfasser beginnt seine Untersuchungen mit den Werken der „,Vor-bertramischen Zeit“, bespricht dann in einzelnen Kapiteln „Die böhmische Frage und Meister Bertram“, ,Konrad von Soest und die Lübecker Malerei“, ,,Meister Franke, das Norddeutsche und seine Wirkung“ und schließt mit dem Kapitel „Stilperiode des bürgerlichen Realismus“. Die Dissertation von Martens zeichnet sich sowohl durch eine außergewöhnliche Klarheit der Gliederung als auch durch die Weite der kunsthistorischen Sicht aus. Darüber hinaus zeigt sie durch die nachgewiesenen Datierungen nicht nur für die Erforschung der mittelalterlichen Tafelmalerei, sondern auch für die gleichzeitige Plastik neue Wege auf.

Das mecklenburgische Porträt vom Spätbarock bis zum Klassizismus ist Gegenstand einer Untersuchung von Vera-Maria Ruthenberg. In ihrer Dissertation ,Die Entwicklung des Porträts in Mecklenburg von Beginn des 18. Jahrhunderts bis zur Mitte des 19. Jahrhunderts“ (2729) gibt sie eine Zusammenstellung der mecklenburgischen Bildnismaler des späten 18. und frühen 19. Jahrhunderts.

Der Übergang vom 18. zum 19. Jahrhunderts ist in Mecklenburg durch die beiden überragenden Gestalten $\mathrm{C}$. D. Friedrich und $\mathrm{Ph}$. O. Runge bestimmt, denen sich als ähnlich gestimmte Seele der von weniger überragender Größe Georg Fr. Kersting anschließt.

Die Literatur über Caspar David Friedrich hat sich in den letzten 30 Jahren so sehr vergrößert, daß es nicht möglich ist, auf die etwa 80 Titel im einzelnen einzugehen. Seit den um die Jahrhundertwende erschienenen Aufsätzen Andreas Auberts (2923, 2924 u. 2967) wuchs das Friedrich-Schrifttum in den 2oer Jahren rasch an. Das forschende Interesse dieser Zeit an Friedrich ist nicht verwunderlich, da man geistesverwandte Züge in der Romantik und Caspar David Friedrich sah. Als wichtigste Veröffentlichung dieser Jahre sind Otto Fischers Arbeit „Caspar David Friedrich, die romantische Landschaft“ (2969) und W. Wolfradts Untersuchung „Caspar David Friedrich und die Landschaft der Romantik“ (2968) zu nennen. Beide Publikationen sind in ihrer wissenschaftlichen Objektivität noch heute vorbildlich. Die Friedrich-Forschung der zoer Jahre stand hauptsächlich im Zeichen der tendenziösen Interpretation durch die faschistische Ideologie. Aber trotzdem erschienen in dieser Zeit zwei umfassende Arbeiten über Friedrich, die frei von einseitiger Betrachtung sind, Herbert v. Einem: „Caspar David Friedrich“ (2918) und Friedrich Nemitz: „Caspar David Friedrich. Die unendliche Landschaft" (2964). Sie gehören noch heute zu den besten zusammenfassenden Darstellungen der Kunst Friedrichs. Als Studie über eine begrenzte Einzelfrage darf das Heftchen Otto Schmitts über ,Die Ruine Eldena im Werk von Caspar David Friedrich" (2977) genannt werden, die trotz ihrer Kürze in ihrer Eindringlichkeit vorbildlich ist.

Die Beschäftigung mit dem Zeitgenossen Friedrichs, dem in Güstrow ansässigen Maler stiller Interieurbilder Georg Friedrich Kersting, begann ebenfalls um die Jahrhundertwende. Wie bei Friedrich fand diese erste Aufmerksamkeit ihren Niederschlag in Zeitschriftenaufsätzen. Eine gründliche Untersuchung und eine Zusammenstellung des Werkes erfolgte erst 1931 in der Publikation von Oscar Gehrig ,Georg Friedrich Kersting, ein mecklenburgischer Maler aus der Zeit der Freiheitskriege" (3033).

Die Entdeckung Philipp Otto Runges dagegen erfolgte schon um die Mitte des 19. Jahrhunderts mit dem 1868 erschienenen Aufsatz von Theodor Unruh „Philipp Otto Runge“ (3080). Aber ein kontinuierliches Schrifttum begann erst nach der 1933 veröffentlichten Hamburger Dissertation von Otto Böttcher „Philipp Otto Runge. Ein Beitrag zur Kunstgeschichte der Romantik und des Klassizismus“ (3067). 
Aus dieser Dissertation entstand Böttchers für alle spätere Runge-Forschung verbindliche Arbeit (3068). Mit den Briefen und Schriften Runges beschäftigten sich dann Forsthoff, Degner und Ch. A. Isermeyer (3072, 3074 u. 3076).

Neben diesen Großen der Kunstgeschichte haben nur die Maler, etwa gleicher Zeit, Paul Suhrlandt und Gaston Lenthe, eine Würdigung erfahren, die in einigen kleinen Aufsätzen, vor allem in den Mecklenburgischen Jahrbüchern, niedergelegt ist (3048.u. 3128-3132). Die mecklenburgische Kunst des 19. und 20. Jahrhunderts hat, wie in der Architektur und Plastik, auch in der Malerei die kunstgeschichtliche Forschung zu keinen nennenswerten Arbeiten angeregt.

\section{Kunsthandwerk und Volkskunst}

Bedeutende Leistungen kann das mecklenburgische Kunsthandwerk nicht aufweisen. Wie in der bildenden Kunst fehlt es auch hier den Mecklenburgern an Formenphantasie und Ausdrucksmöglichkeit. Das Möbelhandwerk zur Zeit der Renaissance und des Barock in Lüneburg und Schleswig-Holstein, ein wichtiger Zweig des norddeutschen Kunsthandwerks, hat in Mecklenburg keine ebenbürtigen Werke hervorgebracht. Lediglich die Goldschmiedekunst und der Metallguß haben eine vom 14. Jahrhundert bis ins Rokoko reichende Tradition gehabt. Die mittelalterlichen Goldschmiedearbeiten, die über das ganze Land verstreut sind und als liturgische Geräte in kleinen Dorfkirchen aufbewahrt werden, hat K. J. Maercker in seiner Dissertation „Mittelalterliche Goldschmiedearbeiten in Mecklenburg“ (3170) publiziert. Er kommt dabei zu interessanten Ergebnissen über das Goldschmiedehandwerk in den Städten, vor allem in Rostock.

Die mecklenburgischen Glocken und ihre Gießer finden bisher meist nur in kleinen, mehr geschichtlich als kunstgeschichtlich wichtigen Aufsätzen Erwähnung.

Von den Arbeiten über Textilien sind vor allem die zahlreichen Untersuchungen über den CroyTeppich zu nennen (3563-3572). Dieses für die Reformationsgeschichte Pommerns so wichtige Dokument fand wegen seiner ikonographischen Thematik allerdings bei den Historikern mehr Beachtung als bei den Kunsthistorikern.

Von höherer Bedeutung als das Kunsthandwerk ist in Mecklenburg die Volkskunst. Besonders das mecklenburgische Bauernhaus hat die Forschung vielfach beschäftigt (1231-1295a). Gerade die letzten Jahre haben hier durch die Methode der Gefügeforschung viel Neues gebracht (1295a). Aber auch der ländliche Hausrat und die Bauerntracht haben in ihren charakteristischen Formen zu einer großen Anzahl wissenschaftlicher Arbeiten angeregt.

Noch bestehende Lücken in der Volkskunst-Literatur, deren Ausfüllung sehr wünschenswert ist, dürften durch die gegenwärtige Förderung derartiger Forschungsarbeiten bald geschlossen sein. 
